\title{
History, development and trend of Fractal based biometric cryptography
}

\begin{abstract}
This article has been originated to institute for obtaining the History and the trend of Development of Fractal based Biometric Cryptography. Here we endeavour to assemble the bygone information for representing the trend of progress of cryptography operated with the perception of Fractal. On a whole, Fractal is a geometric figure of non-integer dimension that has two properties: First, most amplified images of fractals are approximately identical from the unamplified version, called self-similarity. Second, fractals have fractional dimensions. Barnsley's Iterated Function Systems (IFS) form on the self-similarity of fractal sets can produce the Fractal Image Coding Scheme by using the principle of affine transformation. To encode digital grey level images, Fractal image coding has been used successfully.
\end{abstract}

Keyword: Fractal; Encryption; Fractal image 